\title{
An Annotation System for Egocentric Image Media
}

\author{
Aaron Duane, Jiang Zhou, Suzanne Little, \\ Cathal Gurrin, and Alan F. Smeaton \\ Insight Centre for Data Analytics, Dublin City University, Dublin, Ireland \\ aaron.duane4@mail.dcu.ie \\ \{suzanne.little, cathal.gurrin, alan.smeaton\}@dcu.ie
}

\begin{abstract}
Manual annotation of ego-centric visual media for lifelogging, activity monitoring, object counting, etc. is challenging due to the repetitive nature of the images especially for events such as driving, eating, meeting, watching television, etc. where there is no change in scenery. This makes the annotation task boring and there is danger of missing things through loss of concentration. This is particularly problematic when labelling infrequently or irregularly occurring objects or short activities. To date annotation approaches have structured visual lifelogs into events and then annotated at the event or sub-event levels but this can be limited when the annotation task is labelling a wider variety of topics - events, activities, interactions and/or objects. Here we build on our prior experiences of annotating at event level and present a new annotation interface. This demonstration will show a software platform for annotating different levels of labels by different projects, with different aims, for ego-centric visual media.
\end{abstract}

Keywords: lifelog, annotation

\section{Introduction}

One of the most intriguing forms of lifelog data which people can accumulate is visual data from wearable cameras, recording either continuous video or frequent images taken at regular intervals [2]. This is now referred to as ego-centric image media, reflecting the fact that it is usually taken from the first person viewpoint and can suffer from image quality issues due to camera shake because of movement of the wearer. For the most part, such visual lifelogs are indexed and become searchable based on their metadata, the date, time and perhaps location at which the images were taken. Increasingly we are realising that in addition to this we need to analyse and index visual lifelogs based on content using either manually annotated tags, or automatic detection of semantic concepts. For either of these approaches there is a need for a software tool to allow end users to manually annotate lifelog images, either to train a machine learning classifier to recognise concepts, or to be used directly as lifelog descriptors.

In this paper we introduce a software platform for manual annotation of visual lifelogs. A short demonstration video can be seen at https://goo.gl/ 
ru2ZxZ. We describe the system in terms of the different kinds of user roles involved in the annotation, and then we describe the annotation process itself. This is followed by a short user feedback and evaluation of the interface looking at how it supports different annotation strategies and the requirements of the system.

\section{Annotation System}

\section{$2.1 \quad$ Users}

The annotation system consists of three different user types, the uploader, the annotator and the administrator (or assigner). The uploaders role is to be the camera wearer, the source of the lifelog data that is to be annotated within the system. Uploaders load their data to the database and the system in turn automatically segments the data into 'photo packages'. Upon logging in, the administrator has two primary roles, creating new annotators and assigning annotators to photo packages which have been uploaded. From the annotator's control panel the administrator can see a list of all annotators created within the system. The administrator has the option to edit or delete an annotator via this interface and to view the total number of images an annotator has been assigned to annotate. On selecting an annotator's assign button, the administrator is brought to the assignment interface where they see a list of all photo packages uploaded to the system.

It is important to note that multiple annotators can be assigned to the same photo package simultaneously. This is useful if annotators specialise or more than one annotator is needed to divide the workload of a large photo package. During the development of the system, the requirement for separate annotations from different projects or perspectives, yet on the same photo packages, became apparent. For example, lifelogs may be annotated by a group interested in food intake while another set of annotators may be interested in the wearers' exercise habits. To address these requirements, the concept of a project was introduced. When assigning an annotator to a package, it is necessary to choose from which project they are being assigned to. This means that different annotators can work on the same photo package under different project guidelines and there will be no risk of interfering with another project's annotations.

When the annotator is ready, they simply press the annotate button next to the package assigned to them and are immediately taken to the primary annotation screen (see Fig. 1). This annotation interface contains three sections, the calendar, the ontology and the annotatable images. The calendar is used to navigate between different days within a photo package if a package spans multiple days. These days are in turn segmented into hours for the purpose of navigability and convenience. When the annotator has targeted the day and hour they want to work on, they can begin selecting the photos they wish to annotate. In Fig. 1 we can see, from the blue border surrounding the images, that currently 10 images have been selected for annotation. 
The annotation system imposes a 3 level hierarchical ontology of terms with terms being selectable via a one-click concertina navigation on the left side of the screen. Level 1 of the ontology appears in blue, level 2 in orange and level 3 in white. At this point the annotator will navigate through the ontology and choose which leaf node annotation terms they wish to attach to the selected images. Upon choosing an annotation term, the selected images are highlighted with a green overlay to indicate that they have been annotated. In the top left corner of each image, the annotator can see how many terms are currently attached.

After adding a term to a single or to a group of images, the annotator can remove the annotation by clicking on the term a second time in the ontology. For this option to be available, all selected images must contain the annotation already, otherwise clicking on the term in the ontology will simply add the annotation to any images that do not already have it.
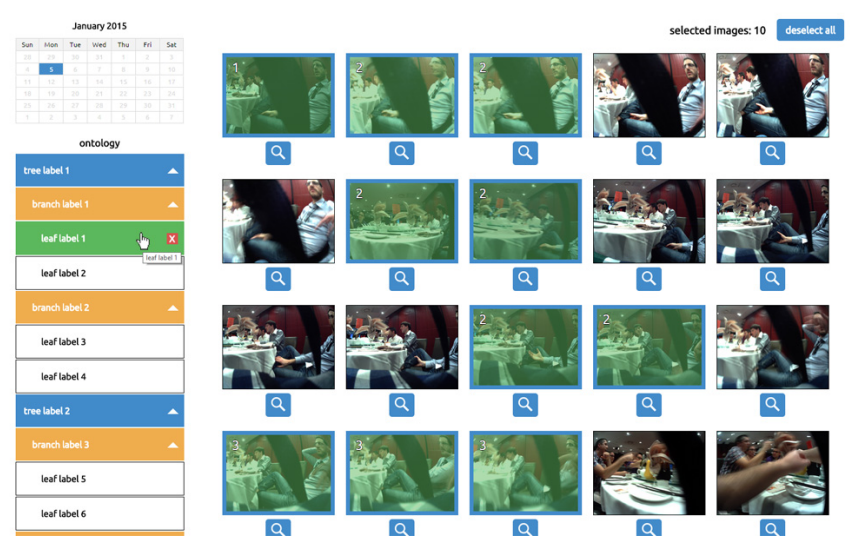

Fig. 1: Annotating images using the ontology.

If the annotator needs to examine an image closely or to see a list of all annotations attached to an image, they have the option of clicking the magnifying glass beneath each image. Upon doing so, an overlay will appear over the screen containing a larger version of the image and beneath that, all the annotation terms attached to this particular image (Fig. 2). It is important to note that the annotations from all projects and all annotations appear in this overlay. The annotator cannot interact with another projects annotation terms and they do not count toward the total terms attached to the image within their project.

The visibility of other projects in the annotation space was introduced because it is often beneficial for an annotator to see what other annotation terms different projects have attached to an image. If this is not the case, the annotator can elect to ignore other terms and focus on their own annotations which are highlighted in green and which they have the option to remove. If the annotator needs to see a full resolution version of the selected image, they can click the 


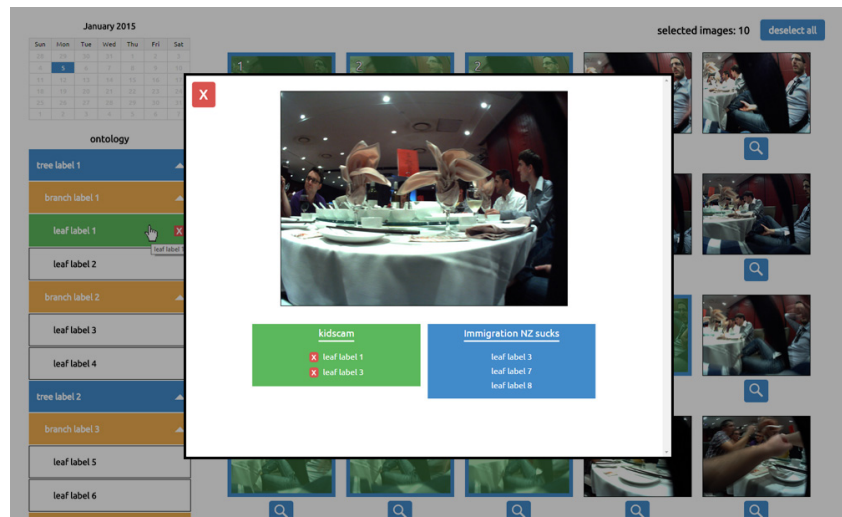

Fig. 2: Exploring details of a single image.

image from within this overlay and a new tab will open containing the image at its native resolution.

\section{Demonstration proposal}

The proposed demonstration will use data gathered live at the conference and engage with the users to rapidly annotate sample data. Users will gain an understanding of the unique requirements for annotating lifelog data and how this may be used in a variety of applications. In addition the demonstration will provide the opportunity for further observations as to the annotation strategy and possible future improvements for the interface.

The annotation tool introduced in this paper offers an alternative to the lifelog browser tool described in [1] and over which it has several advantages including a more flexible framework to ingest and annotate with structured levels of labels and enabling multiple annotations of images based on individual projects. Labelling ego-centric visual media for both information retrieval or higher-level data analytics at the event, activity and object level will influence the annotation strategy and hence the future development of specialised annotation interfaces.

\section{Acknowledgements}

This paper is based on research supported by Science Foundation Ireland under grant number SFI/12/RC/2289 and Health Research Council of New Zealand.

\section{References}

1. A. R. Doherty and A. F. Smeaton. Automatically segmenting lifelog data into events. In Image Analysis for Multimedia Interactive Services, 2008. WIAMIS'08. Ninth International Workshop on, pages 20-23, 2008.

2. C. Gurrin, A. F. Smeaton, and A. R. Doherty. Lifelogging: Personal big data. Foundations and Trends in Information Retrieval, 8(1):1-125, 2014. 